Archives de sciences sociales des religions

116 | octobre - décembre 2001

Varia

\title{
Conversion, tradition, institution
}

\section{Christian Décobert}

\section{OpenEdition}

Journals

Édition électronique

URL : http://journals.openedition.org/assr/541

DOI : 10.4000/assr.541

ISSN : $1777-5825$

\section{Éditeur}

Éditions de l'EHESS

\section{Édition imprimée}

Date de publication : 2 octobre 2001

Pagination : 67-90

ISBN : 2-222-96712-0

ISSN : 0335-5985

Référence électronique

Christian Décobert, "Conversion, tradition, institution », Archives de sciences sociales des religions [En ligne], 116 | octobre - décembre 2001, mis en ligne le 17 octobre 2005, consulté le 03 mai 2019. URL : http://journals.openedition.org/assr/541; DOI : 10.4000/assr.541 
Arch. de Sc. soc. des Rel., 2001, 116 (octobre-décembre) 67-90

Christian DÉCOBERT

\title{
CONVERSION, TRADITION, INSTITUTION
}

\author{
À propos de : \\ ALTEMEYER (Bob), HUNSBERGER (Bruce), \\ Amazing Conversions. Why Some Turn to Faith \& \\ Others Abandon Religion, New York, Prometheus \\ Books, 1997, 268 p. (bibliogr.). \\ LAMB (Christopher), BRYANT (M. Darrol), éd., \\ Religious Conversion. Contemporary Practices and \\ Controversies, Londres, Cassel, 1999, 342 p. (index). \\ GODO (Emmanuel), s.d., La Conversion religieuse, \\ Paris, Imago, 2000, 332 p.
}

Les ouvrages traitant de la conversion religieuse ne manquent pas, ces derniers temps. Comme précédemment ceux sur l'identité et l'ethnicité, ils répondent à l'évidence à une demande sociale diffuse, à une interrogation politique peu articulée. Les dits risques sectaires, ruptures de transmission des grandes traditions religieuses, nouvelles formes de communalisation devraient, en effet, faire l'objet de constats pratiques auxquels les spécialistes du religieux, les sociologues, les historiens sont sensés apporter quelque matériau - dans le respect de leur discipline. La présente note critique considère trois ouvrages récents, dans la mesure où, ensemble, ils paraissent représentatifs du champ des intérêts savants sur ces questions très contemporaines.

La Conversion religieuse est un ouvrage collectif, dirigé par Emmanuel Godo. Il est constitué de deux parties, d'inégales longueurs sinon d'inégales importances. La majorité des contributions, en effet, traitent de la conversion religieuse en littérature, entendue soit à partir de récits de conversion tels qu'ils apparaissent dans des textes littéraires modernes, soit comme des conversions de littérateurs. Ces contributions sont précédées par des études plus historiennes, qui travaillent, dès son origine, la question de la conversion au (et dans le) christianisme.

Religious Conversion, autre ouvrage collectif, édité par Christopher Lamb et M. Darrol Bryant, se donne pour horizon, par des approches diverses mais toujours croisées, de répondre à quelques lancinantes questions: de quoi parle-t-on, aujourd'hui, quand on parle de conversion? Et de quoi parlait-on avant? Est-ce 
que la conversion, comme changement du sens de la vie, n'est qu'un phénomène chrétien? Que dire de la dramaturgie des récits de conversion ? Est-ce que le retour au religieux est une conversion? Trois sections construisent l'ouvrage (théorie de la conversion; signification de la conversion dans les différentes religions; tensions entre les traditions à propos du phénomène de conversion), pour, en définitive, tenter de comprendre s'il y a un sens commun à la conversion.

Le troisième ouvrage, Amazing Conversions, est, quant à lui, le rapport d'une enquête. Son objet est d'appréhender comment les acteurs se représentent leur expérience, lorsqu'ils adhèrent à une foi ou qu'ils abandonnent une croyance, en l'occurrence l'une des Églises chrétiennes. L'enquête a été faite auprès de plus de 4000 étudiants (de première année) de deux universités canadiennes (Manitoba University, Winnipeg, Manitoba et Wilfred Laurier University, Waterloo, Ontario). Il s'agit d'un milieu de tradition majoritairement protestante mais où les catholiques constituent une minorité importante (un petit tiers). Il ressort que 1,4\% des étudiants interrogés sont devenus incroyants (Amazing Apostates: AAs) et que $0,8 \%$ se sont convertis, devenant croyants après avoir été incroyants ou indifférents et vivant dans un milieu incroyant (Amazing Believers: ABs) ; AAs et ABs déclarant explicitement être passés d'un système de pensée à un autre plutôt que d'une religion à une autre. Ils sont «amazing », étonnants, dans la simple mesure où ils détonnent, où ils s'opposent au monde qu'ils ont reçu en héritage - le qualifiant n'indique, en l'occurrence, qu'un écart, et non un jugement.

Un parcours de ces trois ouvrages permet précisément d'en dire un peu sur ce qui gouvernait le projet de Christopher Lamb et M. Darrol Bryant: en quoi la conversion religieuse est chrétienne ? pourquoi la conversion chrétienne est la scénographie d'un drame ? et qu'en est-il aujourd'hui de ce drame? De telles questions sont intimement liées entre elles.

\section{La conversion inversée}

L'enquête me servira de fil. Les AAs disent avoir accordé une grande importance aux interrogations touchant les sources de l'autorité religieuse, en revanche ils sont peu attirés par ce qui relève de l'orthodoxie chrétienne, de ce qu'il y a à croire. À l'inverse, les ABs font montre d'un très faible intérêt pour ce qui relève de l'autorité religieuse mais d'une forte attirance pour ce qui touche au domaine de l'orthodoxie chrétienne.

Les AAs sont au nombre de 46. Il y a nettement plus de garçons que de filles, alors que le ratio général des étudiants de ces universités est de 6 filles pour 4 garçons. La moitié des AAs sont des premiers-nés, et $69 \%$ d'entre eux viennent de familles catholiques, alors que les catholiques ne sont que $29 \%$ du corpus.

Les enquêteurs expliquent ce nombre important de AAs issus de familles catholiques simplement par le fait que ceux-ci, en tout cas dans les milieux anglo-saxons, imposent une éducation religieuse plus rigide que les protestants, de même qu'ils diffusent un enseignement très aliénant, particulièrement sur les questions de sexualité. Rigidité, aliénation : c'est de cela, semblerait-il, que les AAs 
cherchent à s'éloigner, à se libérer. Nombre de récits de ces cheminements signalent qu'un sentiment de culpabilité apparaît au moment des premiers doutes religieux, alors même que les réponses aux questionnements, les affirmations face à leurs doutes leur semblent toujours plus insatisfaisantes au sein du milieu familial. La peur et la culpabilité sont mêlées. Mais ces mêmes récits opèrent une certaine distanciation par rapport à cette période-là, laquelle est considérée comme un temps de naïveté : une distanciation qui révèle un fort sentiment de discontinuité dans l'existence.

Les causes déclarées au malaise et aux questionnements sont très rarement le conflit ou la mésentente (il n'y a pas de problème grave avec les parents, par exemple) : ces causes sont les doutes religieux eux-mêmes. Ainsi, les raisons de l'apostasie seraient, selon les étudiants interrogés, dans les croyances religieuses elles-mêmes, dans le peu de crédit qu'on peut leur accorder, et non dans quelque cause indirecte, plus ou moins masquée. Les AAs ne mentionnent pas d'intermédiation à leur incroyance - quelqu'un qui les aurait aidés ou incités à abandonner leur croyance. Le coût de l'apostasie est considéré comme important: isolement et confusion; le gain pour l'acteur est d'avoir enfin trouvé sa propre identité. Mais pourquoi eux ? se demandent les auteurs. Ce sont des jeunes gens qui refusent de se voir dicter ce qu'ils doivent croire, qui ressentent l'impossibilité de croire. Les AAs sont toujours d'une grande exigence, ils sont d'ailleurs de brillants sujets au plan scolaire, et s'il y a doute sur un point d'autorité religieuse, c'est le système de vérité qu'est la religion qui s'effondre entièrement - en ce sens, ils comprennent la cohérence de ce qu'on leur a enseigné : la religion est un tout.

La plupart des ABs disent avoir eu des problèmes personnels ; 1'abus d'alcool étant le plus courant des symptômes évoqués. Comment sont-ils devenus religieux ? Ils y furent à peu près toujours conduits par des pairs, des camarades de classe, des amis de leur génération. Ils n'évoquent pas de faits de médiation scripturaire ou rituelle, comme par exemple une lecture de la Bible, qui aurait provoqué le processus de conversion. Généralement, ils disent avoir «atteint un point » où ils ont accepté en bloc toutes les croyances chrétiennes - il n'y en a pas une qui aurait servi de déclencheur aux autres. Pour décrire la découverte religieuse, l'idée souvent émise est celle de soudaineté, de révélation, de "flash », bien qu'ils avouent avoir été «en recherche ». Le sentiment de peur et de culpabilité est peu présent chez les ABs, beaucoup moins que pour les AAs ; ils ont au contraire l'impression que leur milieu familial a assez bien accepté leur décision. Quant à la dénomination religieuse actuelle qu'ils se donnent, la plupart se disent globalement «chrétiens » mais ceux qui précisent une affiliation particulière mentionnent toujours un groupe «fondamentaliste ». La dramatisation du changement que la conversion a suscitée est grande, ce qui crée une distanciation forte avec l'état antérieur de non croyant. Nulle révolte n'est, cependant, relatée, nul conflit au sein de la cellule familiale, mais un sentiment plutôt vague de mal être ancien. Les ABs sont dans l'ensemble moins brillants, en classe, que les AAs.

Le coût de la conversion semble relativement faible, en revanche le gain est dit immédiat : c'est la sécurité qui vient en premier à l'esprit («Dieu me protège, me protègera »), une sécurité qui se prolonge dans la certitude d'accéder au paradis après la vie ; viennent aussi la satisfaction, le contrôle des émotions, et le sentiment de l'improbabilité d'un retour en arrière. Au contraire, le prix à payer des AAs est visiblement plus lourd, car les regrets (d'avoir quitté une croyance familiale) sont 
sérieux. Les AAs se sentent indépendants, les ABs se sentent membres d'un groupe puissant. Les AAs se disent forts, les ABs savent qu'ils étaient faibles. Consciemment, les AAs ont abandonné tout ce qui attire les ABs : sécurité, guidance, solidarité, vie après la vie.

Les deux actes, conversion et apostasie, ont été posés, dans la facture même de l'ouvrage, dans une parfaite symétrie. Il est aisé d'adhérer à la foi chrétienne, beaucoup moins d'y renoncer, lorsqu'on est né dans un milieu majoritairement chrétien. D'où les traits marqués qui distinguent les apostats (étudiants brillants, sentiment d'être forts), traits symétriques de ceux qui dépeignent les convertis (moins brillants, sentiment d'avoir été faibles), comme sont symétriques les éléments de narration de l'apostasie et de la conversion : apostasie dans la douleur, la solitude, après de longues interrogations mais sans une crise que l'on puisse situer avec précision ; conversion dans la félicité, médiatisée par un pair, après une crise personnelle, une sorte de recherche mais surtout le «flash» de la découverte de la vérité.

Le terrain, tel qu'il est présenté, est celui d'une nébuleuse religieuse dominante, le christianisme ; dans ce contexte, le converti rejoint l'un des groupements de cette nébuleuse dominante (Église catholique, United church, Église anglicane, etc.), alors que l'apostat quitte l'un de ces groupements pour ne rien rejoindre ; il n'adhère à aucun autre groupement, serait-il minoritaire. Par ailleurs, il est question d'avantage, de gain à s'être converti, comme il est question de remords et de confusion à avoir apostasié : les termes de la rationalité de l'action et de la déviance s'imposent.

Reconsidérons en ces termes les données de l'enquête. Le gain du converti est tangible : il parle de sécurité, de protection. Le changement de la conversion est que désormais Dieu le protège, le protègera jusque dans l'au-delà : le groupement dans lequel il est entré le protège, avec ses frontières, ses certitudes. Au demeurant, le converti accorde une foi très égale à un corpus d'une orthodoxie sans faille. Rétroactivement, le temps d'avant la conversion est considéré comme celui de l'insécurité, de l'état de faiblesse, de fragilité. Le mot « fragile » revient dans les récits, et l'un des symptômes déclarés de cette fragilité est le fait que le futur converti ne pouvait contrôler ses émotions, qu'il succombait à ses passions, et notamment qu'il tombait dans l'alcoolisme. En revanche, le temps présent est celui de la domination de soi. Une domination de soi perçue comme autonomie, à l'inverse de l'hétéronomie passée. Après avoir eu lieu, donc, l'action de conversion est relatée comme très rationnelle, où le bénéfice est évident mais où « l'offre » qui est faite par le groupement religieux d'accueil (telle obédience chrétienne) ne correspond qu'à une valeur (la sécurité) aussi vague qu'universelle (la protection offerte par une institution à caractère communautaire).

D'autre part, l'alcoolisme (comme l'usage de la drogue...) est une déviance sociale, reconnue par l'acteur comme telle. Mais le récit de la conversion propose un discours religieux de la déviance sociale pour lequel l'alcoolisme est vu comme une première manifestation d'anomie, à la fois comme un défaut d'intégration à tout groupement porteur de valeurs sacrées et comme un comportement qui mène à la marginalisation sociale. Le récit se construit ainsi sur un schéma qui situe le passage au religieux : considérée comme une anomie, la déviance sociale constitue par là même une déviance religieuse. Le «flash », c'est-à-dire la découverte soudaine de la foi, provoque un besoin de conformité religieuse immédiate (une irréprochable orthodoxie), il fait entrer le converti dans le monde de l'autonomie par inté- 
riorisation, acceptation, accessibilité de ce qui le règle - l'altérité du référent divin se dissout dans la reconnaissance pour soi d'une théonomie, laquelle supporte le retour à la conformité sociale. C'est bien le jugement qui constitue le moment du travail du groupement religieux influent, au sens logique de jugement, autrement dit de faculté de mettre en relation d'équivalence un fait social et un précepte religieux - où le prédicat est ce qui relève du religieux (l'alcoolisme est un péché). La conversion (tout au moins telle qu'elle est représentée ici) est une affaire de « réinsertion », elle a pour origine une crise et comme fin une guérison, elle est dans le même temps un passage par l'évaluation des actes de la personne en crise sur l'échelle des normes d'un groupement influent.

L'apostasie pose d'autres questions. Elle semble ne faire suite à aucune crise déclarée, mais plutôt à un sentiment sourd d'insatisfaction. Une insatisfaction entretenue par des réponses décevantes à des questions directement religieuses que (se) posait l'acteur. Ces questions relèvent du domaine de l'autorité religieuse, puisque précisément l'acteur est issu d'un milieu où, beaucoup plus que de moyenne, une emphase est portée sur les faits d'autorité religieuse. Mais comment définir ce que les auteurs entendent par "autorité religieuse »? Ou plutôt, comment mettre en rapport les deux pôles sur lesquels l'enquête repose largement, autorité et croyance? L'autorité d'une chose est ce qui fait qu'on la croit véridique ; l'autorité est également la distinction d'une personne en vertu de laquelle on la croit. Si dans un milieu où l'attention est forte pour ce qui fait autorité, si l'acteur (le candidat à l'apostasie) est insatisfait par le discours religieux qu'il entend, c'est qu'en réalité il exprime une méfiance envers les personnes autorisées. Les sources de l'autorité religieuse ne lui semblent pas actualisées chez ou par les personnes autorisées. N'est-il pas d'ailleurs révélateur que les apostasies ont, proportionnellement, surtout lieu en milieu catholique ? N'est-ce pas parce que c'est dans le catholicisme anglo-saxon que le dispositif autoritaire est le plus prégnant ?

Ainsi l'apostasie serait la conséquence d'une dénonciation froide, sans conflit réel, du dispositif au nom même de ce que celui-ci offre à croire. L'incroyance serait, si nous suivons les auteurs de cette enquête, comme le constat de l'absence d'ascendant de ce ou de celui qui est à croire.

Les ABs gagnent une sécurité, les AAs gagnent une identité. Les deux expériences sont-elles symétriques, comme le plan même de l'ouvrage voudrait le signifier? L'efficacité de la conversion est ici à la fois le retour à la conformité sociale et l'intégration sociale, l'un et l'autre étant les deux faces d'un même processus. Le converti a la sensation légère d'avoir gagné quelque chose. Et l'apostat? Le rejet du dispositif autoritaire s'accompagne d'abord, pour lui, de lourds remords, et plus tard d'un sentiment de culpabilité, de confusion. Il a bien agi, mais il a aussi mal agi. Ne serait-ce que très indirectement, l'apostat sait ou croit savoir qu'il a été mis en position de déviant et il a accepté de l'être. Il a intériorisé sa déviance ${ }^{1}$ et cette intériorisation est d'autant plus forte que l'auto-reconnaissance de la déviance se produit en l'absence de conflit ouvert. Le stade suivant de l'expérience de l'apostasie caractérise alors l'incroyant dans un milieu où le christianisme liturgique et communautaire reste prégnant: c'est la posture de la déviance stable.

${ }^{1}$ Voir Howard S. Becker, Outsiders. Études de sociologie de la déviance, Paris, Métailié, 1985 ; Norbert Elias \& John L. Scotson, Logiques de l'exclusion, Paris, Fayard, 1997. 
Il y a deux modalités possibles d'évolution de l'apostasie. La première est l'enclenchement d'une conversion sur l'apostasie. L'apostat quitte la religion familiale et, après une période plus ou moins longue et tourmentée, entre dans un mouvement religieux quelconque. Il se trouve dès lors dans la situation décrite plus haut où l'anomie se retourne en une hypernomie, où le doute lui-même est constitutif de son cheminement vers le salut offert par le mouvement religieux d'accueil. Le remord, c'est-à-dire l'acceptation d'avoir péché, devient un motif de délivrance, l'acteur accède à une nouvelle vie, c'est un born again. Mais les apostats interrogés dans cette enquête ne se retrouvent pas dans une telle instance, dans la simple mesure où ils offrent une très faible ouverture à l'autoritarisme, en d'autres termes, où ils sont très peu attirés par les figures d'autorité charismatique, les prophètes, gourous et autres prédicateurs.

La deuxième modalité d'évolution de l'apostasie est le fait de persévérer dans l'incroyance. Et de s'y trouver bien. Mais les symptômes décrits chez les AAs ne correspondent pas non plus à cette quiétude. Ceux-ci vivent dans la confusion, en d'autres termes, ils sont dans l'impossibilité de sortir de la position d'anomie à laquelle on les a et ils se sont assignés et ils sont incapables d'accéder à un état d'autonomie. L'autonomie étant en l'occurrence l'état dans lequel l'on pense une loi pour soi à partir de ce que l'on est ; la confusion est le contraire de l'autonomie, c'est un désordre qui dure.

Sujet brillant, réfléchi, l'apostat de la présente enquête s'attribue lui-même les traits d'un «carriériste» solitaire de la déviance, mais il dit également qu'il y trouve - enfin - une identité. Ce qui veut dire que l'identité consiste pour lui à être semblable à ce qui est opposé à ce qu'il a été. Cette identité, il convient de l'appeler une antinomie, c'est-à-dire à la fois de l'autonomie et de la confusion, un état de contradiction assumée des règles. Entre la carrière de l'incroyance déviante (anomie) et celle de l'incroyance valorisée (autonomie), il y a celle, suspendue, de l'incroyance honteuse.

Voilà donc un discours de la conversion qui est essentiellement un discours de la discipline (du nomos) et de l'identité, et de leur lien mutuel. Mais il convient de renverser la perspective des enquêteurs. Un constat a été fait et qui ne peut que servir de prémisse à toute enquête sur la conversion aujourd'hui, en Occident dit chrétien : les mouvements, massifs ou ostensibles, de conversion se développent dans un contexte de forte dérégulation du croire ${ }^{2}$. Les conversions sont les conséquences à la fois les plus paradoxales et les plus attendues de cette dérégulation, nous ne pouvons plus guère ignorer ce fait.

Les enquêteurs, qui semblent avoir travaillé dans un état d'empathie avec les étudiants qu'ils interrogeaient, n'ont pas explicitement fait le constat. Pourtant les faits sont là. Dans l'enquête, la conversion est vue comme une aspiration à la sortie de l'anomie, comme un cheminement vers une sécurité en quelque sorte vacante (puisqu'il n'y a pas de discours du salut), une sécurité qui n'est pas articulée sur un attachement à l'autorité qui donne à croire (laquelle est l'actualisation d'un dogme - ce par quoi un dogme tient son efficacité) mais sur une parfaite orthodoxie des croyances (lesquelles sont les énoncés d'un dogme dans une tradition héritée). En

2 Frédéric Gugelot, La conversion des intellectuels au catholicisme en France 1885-1935, Paris, CNRS Éditions, 1998 (cf. Arch. 108.49) ; Danièle HERVIEU-LÉGER, Le pèlerin et le converti. La religion en mouvement, Paris, Flammarion, 1999 (cf. Arch. 108.50). 
l'occurrence, le converti dissocie les termes qui, dans leur rapport obligé, doivent instaurer la croyance : d'un côté ce qui est donné à croire, de l'autre, la voie par laquelle cela est donné à croire. Nous le savons, c'est bien ce rapport obligé qui est aujourd'hui défait ${ }^{3}$.

C'est pourquoi il faut accorder de l'importance à la remarque des enquêteurs selon laquelle les ABs sont en quelque sorte attirés par l'autoritarisme et rejoignent plus naturellement les groupements religieux dits fondamentalistes. De tels groupements sont certes ceux qui prétendent retourner à un christianisme originaire, mais surtout ce sont ceux qui imaginent et tentent de recréer un christianisme dominant. Leur diagnostic est qu'une irrémédiable perte civilisationnelle se conjugue avec la dérégulation du croire chrétien et ils agissent pour restaurer les règles dominantes de ce croire chrétien. De fait, cette tendance est cohérente avec nombre de récits.

À l'inverse, on pourrait dire que l'attitude de l'apostat honteux consiste à identifier ce désordre qui frappe la croyance. L'apostat honteux affronte la question de l'autorité qui donne à croire et, en l'absence de réponse (de constat d'une efficacité de la croyance), il s'installe dans une antinomie inconfortable, toute empreinte d'une nostalgie récurrente pour l'état de l'homme soumis aux règles de l'énonciation de ce qui est donné à croire. L'un avec l'autre, le converti et l'apostat de cette enquête rendent compte d'une situation de grippage d'une mécanique, où les biens de salut sont là mais comme figés, et où la reproduction de ces biens ne semble plus se faire. Les conditions d'élaboration d'une norme actuelle semblent ne plus être réunies au sein des grands groupements religieux.

Mais la faute de l'apostat n'est pas l'anomie, l'apostat est déviant sans être anomique. La culpabilité ressentie n'est pas celle d'un homme qui a péché en acte mais qui a un défaut de foi, qui ne peut plus croire. S'il se place effectivement en marge d'un monde croyant, c'est dans la seule mesure où il radicalise l'état de dérégulation du croire, où il contribue à cette dérégulation : s'il n'a plus de repère d'autorité de ce qui est donné à croire, c'est parce qu'il fait le diagnostic que son milieu religieux d'origine n'a plus l'armature qui fait les groupements religieux. Diagnostic d'une perte: il a dans le même temps le regret d'un monde réglé. L'apostat antinomique est coupable de nostalgie.

C'est en définitive la figure de l'apostat qui permet, dans sa nostalgie même, de saisir ce que pourraient être les termes de la conversion religieuse. L'apostat est en quelque sorte affronté à un certain schéma, à quelque chose qu'il voudrait inverser. Ce schéma est narratif.

${ }^{3}$ Voir notamment Raymond Lemieux et Micheline Milot, s.d., Les croyances des Québecois. Esquisses pour une approche empirique, Cahiers de recherches en sciences des religions 11, Québec, Université Laval 1992 ; le dossier des Arch..83 (1993) sur la religion aux États-Unis et surtout David A. Roozen \& Jackson W. CARroll, Wade C. Roof, «La génération née après-guerre et la religion instituée. Un aperçu sur 50 ans de changement religieux aux États-Unis », Arch. 83, 1993, pp. 25-52. 


\section{La conversion est un récit}

La conversion, du moins au christianisme, s'exprime dans un récit. Mais parler des récits de conversion semble ne mener à rien, tant ceux-ci sont convenus, se ressemblent tous, semblent décalés par rapport à la situation sociale de ceux qui les racontent. La répétition à l'envie d'un semblable matériau est plus le signe de sa pauvreté que de sa richesse. Les histoires individuelles se moulent dans «un schéma très classiquement attesté, qui oppose un " avant » tragique, désespérant ou simplement médiocre, et un "après » caractérisé au contraire par la plénitude du sens » ${ }^{4}$. Et le sociologue ou l'historien l'invoque, bien souvent, pour d'autres objets que le fait de conversion : les faits de déculturation, la déviance sociale, les stratégies des groupes dominants, des «minorités actives», etc. Pour autant, la stabilité d'un récit que l'on dit tellement convenu fait problème : s'il y a convention, d'où vient-elle ? Autrement dit, si une convention gouverne une certaine forme de présentation de la conversion, il faut bien que cette convention, pour exister, tire sa légitimité de quelque chose qui permet son actualisation - et ce quelque chose doit avoir pour nature un régime institutionnel.

Du récit de la conversion au christianisme à certaines institutions chrétiennes, tel est précisément le parcours, à rebours, que les deux autres ouvrages commentés ici (La conversion religieuse, Religious Conversion) permettent d'entreprendre. Mais en regard, et comme une expérimentation par la négative, les Amazing Believers et Amazing Apostates, dans l'inaccomplissement de leur geste, marquent le défaut de ce régime institutionnel et il faudra y revenir.

Un récit, donc. Beaucoup d'ABs évoquent un passé alcoolique. Plus largement, très nombreux sont les récits de conversion qui évoquent un moment initial de crise personnelle, crise dont les symptômes sont des attitudes de désordre personnel, tels que l'alcoolisme, la toxicomanie, la violence, la promiscuité sexuelle, l'errance ${ }^{5}$.

Or ces délinquances ne sont précisément pas prises (interprétées, narrées) comme des symptômes de désordre social - relâchement des valeurs communautaires, crise de l'autorité traditionnelle, acculturation/déculturation, changement drastique de mode de vie, etc. - que subirait le futur candidat à la conversion. Comme dit justement Patrick Williams, le converti, dans l'évocation de son passé, fait l'inverse du sociologue ${ }^{6}$. Ce qui revient à dire que le converti, dans son récit, se pose en acteur et non en patient (subissant les effets d'une crise à lui extérieure). Cette autonomie de sujet qu'il revendique à la fin de son cheminement, par le choix intime de se convertir, il la pose de fait au début. La crise qu'il a vécue n'est pas un reflet, un effet, elle est au propre une désimplication sociale. C'est sa crise qui identifie l'acteur comme singulier. Cet acteur singulier se raconte.

${ }^{4}$ Danièle Hervieu-LÉGer, Le pèlerin et le converti, op. cit., p. 131.

${ }^{5}$ Voir, pour un bon exemple : Patrick Williams, « Le miracle et la nécessité : à propos du développement du pentecôtisme chez les Tsiganes », Arch. 73, 1991, p. 91.

6 Idem, p. 90. 
Il se raconte dans un récit stéréotypé. Lewis R. Rambo et Charles E. Farhadian situent en sept moments la logique narrative de la conversion ${ }^{7}$. Et puisqu'il s'agit de la logique d'un processus, les auteurs nomment celui-ci plutôt converting - actif de l'action se faisant - que conversion. Ce modèle, il faut le dire en préalable, ressemble fort à celui que John Lofland et Rodney Stark avaient élaboré dans un célèbre article de $1965^{8}$, à propos de conversions à la communauté des World Savers. Une comparaison entre les deux schémas s'avère utile.

\begin{tabular}{|l|l|}
\hline \multicolumn{1}{|c|}{ Lofland \& Stark } & \multicolumn{1}{c|}{ Rambo \& Farhadian } \\
\hline crise & contexte \\
\hline langage religieux & crise \\
\hline quête & \\
\hline rencontre & quête \\
\hline interaction & rencontre \\
\hline dégagement & interaction \\
\hline engagement & \\
\hline & engagement \\
\hline & conséquences \\
\hline
\end{tabular}

Ce qui importe d'abord est la séquence «crise / quête ». Deux questions se posent à propos de cette séquence. La première est de savoir ce que l'on entend par crise et par quête. La deuxième est d'entrevoir quel est le domaine d'extension d'un schéma de conversion qui repose sur une crise préalable - concerne-t-il toutes les conversions qui se racontent?

La crise est un accident de la vie, un malaise, un mal être, un traumatisme, quel que puisse en être la nature, c'est un moment de désordre. Rambo et Farhadian disent que la quête - qui est une demande religieuse - est une manière de répondre à la crise ${ }^{9}$. La crise serait résolue, ou plutôt déplacée, dans la quête. Mais ne peut-on pas dire que la quête religieuse est une expression de la crise ? C'est ce que suggèrent Lofland et Stark dans une médiation " crise / expression religieuse / quête ». Ils signifient par là que la quête religieuse est l'effet d'une mise en contiguïté d'un sentiment de désordre intérieur et d'un discours religieux dont l'un des thèmes est précisément le désordre et la signification qu'il faut lui accorder. Un langage religieux s'impose ainsi à ce qu'on pourrait appeler un signifiant pur (un sentiment de désordre) pour lui donner un signifié et surtout pour l'articuler sur d'autres signifiés que constituent d'autres éléments du même discours religieux. En conséquence, on ne peut pas prendre la quête religieuse, dans une occurrence traumatique, pour une simple demande dans la mesure où elle se construit plutôt

\footnotetext{
${ }^{7}$ Lewis R. RAmbo \& Charles E. FARHADiAn, « Converting: stages of religious change », Religious Conversion, pp. 23-34.

${ }^{8}$ John Lofland \& Rodney STARK, «Becoming a World-Saver: A Theory of Conversion to a Deviant Perspective », American Sociological Review 30, 1965, pp. 862-875. Cet article n'est pas mentionné par Rambo et Farhadian.

9 « The quest stage encompasses different ways in which people respond to crises and/or the way in which people orient themselves to life, especially the religious life »: Lewis R. RAMBO \& Charles E. FARHADIAN, Religious Conversion, p. 27.
} 
comme un fait de circulation de discours religieux. Une crise quelconque ne devient pas une demande religieuse, mais, sous l'effet de contamination par un discours d'offre religieuse, elle se transmue en une recherche de cette offre.

On pourrait multiplier les exemples d'une telle transmutation. L'un des plus notoires est peut-être celui de la multiplication des conversions au christianisme, dans l'Empire romain, après les périodes d'épidémies, au milieu du III ${ }^{\mathrm{e}}$ siècle notamment ${ }^{10}$. Les épidémies étaient des facteurs puissants de déstructuration sociale, où les familles se fracturaient quand les parents enterraient leurs enfants, où la vie urbaine se dissolvait quand l'espace public était le lieu de la propagation $\mathrm{du}$ mal, quand les citadins fuyaient à la campagne, etc. Or l'une des connotations $\mathrm{du}$ discours chrétien de l'amour du prochain était alors (pour des raisons historiques évidentes, liées aux premières persécutions et au fait que les chrétiens vivaient en petits groupes disséminés) une expression de la forte cohésion sociale. Durant les épidémies, les chrétiens s'activèrent à soigner les malades et à laver les défunts et s'exhibèrent en tant que membres d'une communauté soudée. Le traumatisme de l'éclatement social que pouvaient vivre les survivants païens était ainsi mis en contiguïté avec un discours et une pratique de la cohésion sociale, celle du peuple de Dieu assemblé dans une indéfectible solidarité. Le malheur de nombre d'hommes païens égarés devint, à ce contact, une recherche désespérée de resserrement du lien social - et la réponse chrétienne était là. Mais ce fait, comme d'autres, n'est pas un exemple de marché libre entre une demande et une offre. Bien au contraire, il est celui d'un marché contraint où la demande existe en tant qu'elle est celle qui correspond à une offre présente.

Le schéma proposé par Lewis R. Rambo et Charles E. Farhadian est considéré comme le modèle du récit de conversion. Il me faut commenter cette affirmation implicite. Quels sont les mouvements religieux et les religions que ce schéma concerne réellement? Nombreux ont été et sont les systèmes de croyance à propos desquels une adhésion nouvelle ne se raconte pas, ne fait pas l'objet d'un récit, qui plus est dramatique, avec un parcours qui va d'un mal être, d'un désordre à une identification de soi, à une intégration dans un monde de sens. Il est des religions et des mouvements religieux où une telle conversion dramatique se raconte. Lesquels? Constatons que les trois ouvrages ici présentés ne considèrent que le christianisme et les dits «nouveaux mouvements religieux ».

L'islam est, de ce point de vue du moins, oublié ${ }^{11}$. Or il y a des récits, anciens, de conversion à l'islam, qui se conforment de toute évidence à un certain modèle de narrativité et qui évoquent un moment de crise aiguë, de désordre vécu par le futur converti. De tels récits concernent surtout les compagnons du Prophète, les hommes de la génération fondatrice de l'islam ${ }^{12}$. Mais la crise en question n'est pas celle d'un désordre initial. C'est, bien au contraire, une crise qui est déclenchée, pour l'acteur, par le fait même qu'il se convertit. Et la narration se fait sur un schéma du type : engagement hors de l'islam - rencontre - crise - conver-

10 Voir Rodney StARK, The Rise of Christianity. A Sociologist Reconsiders History, Princeton (N.J.), Princeton University Press 1996, pp. 73-94 (cf. Arch 104.52).

11 Voir Yasin DutTon, "Conversion to Islam: the Qur'anic paradigm », Religious Conversion, pp. 151-165 ; Sophie Gilliat-RAY, «Rediscovering Islam: a Muslim journey of faith », Religious Conversion, pp. 315-332.

12 Christian Décobert, Le mendiant et le combattant. L'institution de l'islam, Paris, Le Seuil, 1991, pp. 125-146. 
sion - engagement dans l'islam. Pour exemple, ceci : 'Umar ibn al-Khattâb est un chef de clan, vaillant guerrier, ennemi de Muhammad et de sa nouvelle croyance mais sa sœur est devenue musulmane - sa fureur contre Muhammad est à son comble mais à mesure qu'il s'approche de lui il devient impuissant, faible, incapable finalement de porter l'épée - il se soumet à Muhammad, il se fait musulman - il sera un chef respecté des armées musulmanes et le deuxième calife de l'islam. La crise est en réalité un moment de la conversion religieuse elle-même, elle ne la précède pas et donc ne la détermine pas. Il s'agit, au propre, d'une conversion vue comme un rite de passage où l'acteur, après une perte de soi dans une épreuve douloureuse, trouve dans l'islam une identité qui lui était déjà promise hors de l'islam.

Le schéma proposé ici est tout autre. Et l'on pourra dire, par hypothèse, qu'il concerne seulement les chrétiens et, par contamination, ceux qui, imprégnés de culture chrétienne, se convertissent à d'autres religions ou mouvements religieux l'islam, le bouddhisme tibétain, le pentecôtisme, l'Église du Christ, l'Église de scientologie, etc.

Un tel schéma se retrouve dans la littérature chrétienne de la conversion. Et je prends le mot littérature au sens plein, pour parler d'écrivains convertis qui rendent compte, en littérature, de leur expérience. Au début du $\mathrm{XX}^{\mathrm{e}}$ siècle, en cette période de siège de la citadelle chrétienne, on assiste à une vague de conversions d'artistes et d'écrivains ${ }^{13}$. Beaucoup ont raconté leur conversion, parfois dans une correspondance privée, souvent comme une œuvre littéraire au sens courant. Une narration stéréotypée s'y retrouve ${ }^{14}$.

Le premier temps de la conversion est donc celui du malaise, du mal être. Mais plus précisément, le mal être est dirigé contre soi, c'est un mécontentement de soi et non d'autrui ou de l'être là. D'entrée (mais rétrospectivement, puisque l'acteur se raconte en écrivant), le candidat à la conversion est un acteur, il n'est pas un patient, il est maître de son destin. Puis vient la quête. Elle est une «quête de réponses ${ }^{15}$, et l'expression de Frédéric Gugelot est significative. L'artiste, ou le littérateur, part en effet à la recherche des différentes réponses, réponses qui font sens au monde - et explications du mécontentement de soi, ce qui est la même chose. Il s'agit en fait pour l'acteur d'épuiser les possibles, d'explorer les systèmes que l'horizon de pensée lui offre, il ne s'agit pas de construire mais proprement d'inventorier le stock des systèmes de pensée, jusqu'à ce que le système chrétien résiste à l'opération d'un inventaire déstructurant. La quête est explicitement une recherche des discours d'offre, qu'ils soient philosophiques, politiques ou religieux. Elle est une demande de contamination par les offres disponibles.

La pensée chrétienne étant là, et non démantelée par l'investigation inquiète de l'acteur-écrivain, vient le moment de la rencontre de l'intermédiateur : un lettré chrétien, un membre d'un réseau d'amitiés, un cercle de discussions ${ }^{16}$. Puis vient l'interaction : l'apprentissage de la liturgie, l'entrée dans le rituel, la découverte de

${ }^{13}$ Frédéric Gugelot, La conversion des intellectuels au catholicisme en France 1885-1935, op. cit. ; voir également, Frédéric Gugelot, « Le temps des convertis, signe et trace de la modernité religieuse au début du $\mathrm{XX}^{\mathrm{e}}$ siècle » à paraître dans les $A S S R$.

14 Frédéric Gugelot, "Les récits de conversion à l'époque contemporaine », La conversion religieuse, pp. 265-267.

15 Idem, p. 266.

${ }^{16}$ Frédéric Gugelot, «Le temps des convertis », op. cit., à paraître. 
l'Écriture. Enfin, la période de dégagement (de la posture ancienne) et d'engagement est marquée essentiellement par la livraison du récit même de la conversion. Un récit qui jette l'opprobre sur les hommes de l'athéisme, sur les politiques de façon générale, dans un discours d'une grande violence parfois ; un récit obligé, également, puisqu'il est le compte rendu impudique d'une expérience d'une grande intimité, mais un compte rendu nécessaire dans la mesure où, comme converti, il faut à l'écrivain porter témoignage. Au total, conclut sur ce point Frédéric Gugelot, le récit se fait œuvre de Dieu ${ }^{17}$, travail de Dieu, Dieu travaille l'homme jusqu'à sa conversion, Dieu travaille le récit en l'inspirant.

L’ouvrage dirigé par Emmanuel Godo dresse un long inventaire de ces expériences littéraires de la conversion, de Max Jacob à Pierre Jean Jouve, de Julien Green à Jean-Claude Renard ${ }^{18}$. Mais le premier des littérateurs convertis qui fit du récit de sa conversion une œuvre fut Augustin d'Hippone, à qui, d'une manière ou d'une autre, chacun d'eux s'oblige à penser.

La conversion d'Augustin est présentée comme «the Christian classic » ${ }^{19}$, le modèle par excellence, parce que les Confessions sont le premier vrai récit d'un cheminement vers le christianisme, vers la vraie foi, avec ses soubresauts, ses lenteurs, ses moments de forte tension, avec l'appareillage psychologique d'un acteur qui regarde au fond de son âme. Le modèle, parce que les Confessions sont également une écriture théologique du fait même de se convertir, au sens où la relation $\mathrm{du}$ processus intrinsèquement continu de la conversion ${ }^{20}$ est à la fois la description de l'œuvre de Dieu dans l'homme et une science de Dieu.

M. Darrol Bryant considère que la conversion d'Augustin se joue sur deux rythmes ${ }^{21}$. Il y a le rythme bref, c'est la révélation dans le jardin de Milan. Augustin ressentait, dit-il, une forte confusion à vouloir et ne pas vouloir Dieu, les larmes d'une forte émotion montraient que cette confusion le secouait totalement. Dans un jardin, il entendit un enfant qui chantait cette comptine : prends et lis... Revenu chez son ami Alypius, il tomba sur la lettre de Paul aux Romains « Point de ripailles ni d'orgies... » ${ }^{22}$. C'est l'expérience du basculement, dans un état permanent de crise liée à un comportement chaotique et à une santé chancelante, au bout d'une quête qui était devenue religieuse; c'est la rencontre soudaine, mais soumise à l'action d'intermédiateurs, avec le texte de l'apôtre qui lui intime l'ordre de se dépouiller de la luxure et de se revêtir du Christ. Ce sera bientôt l'apprentissage du christianisme, de ses rites, de l'Écriture, et son engagement comme clerc et lettré, évêque pourfendeur de l'hérétique. Désordre du comportement et mécontentement de soi ; expression religieuse de ce désordre dans une recherche philosophique du sens des choses; une recherche philosophique qui devient une quête religieuse,

${ }^{17}$ Frédéric Gugelot, «Les récits de conversion à l'époque contemporaine », La conversion religieuse, p. 266.

18 Dans l'ouvrage, les contributions sur les écrivains convertis concernent Max Jacob, Pierre Jean Jouve, Clive Staples Lewis, Aimé Pallière, Julien Green, Lanza del Vasto, Jean-Claude Renard. Celles sur les écrivains qui font de la conversion un motif littéraire concernent La Bruyère, Ulrich Guttinguer, Eugène Sue, Edmond et Jules de Goncourt.

${ }_{19}$ M. Darrol Bryant, "Conversion in Christianity: from without and from within », Religious Conversion, p. 181.

${ }^{20}$ Peter Brown, La vie de saint Augustin, Paris, Le Seuil, 1971, pp. 129-131.

${ }_{21}$ M. Darrol BRYANT, «Conversion in Christianity », Religious Conversion, pp. 181-184.

22 Romains XIII, 13-14. 
dans un désir contrarié de Dieu; la rencontre comme entremise d'amis chrétiens, comme intermédiation d'un enfant chantant, comme parole de Paul qui concerne exactement son état; etc. Les unités narratives évoquées plus bas sont bien là.

Il y a un rythme lent de la conversion d'Augustin, laquelle emplit toute sa vie et n'est circonscrite à aucun moment particulier. Une vie entière de conversion dévoile tout le travail de l'homme à la recherche d'une issue à ce qu'il est et l'œuvre d'un Dieu qui l'offre - insistance est ainsi faite tout au long des Confessions sur les fautes, sur l'impossibilité de trouver la vraie voie en même temps que sur la continuité du cheminement vers elle. Lent cheminement d'une vie gouvernée par le désordre, scansion d'expériences de Dieu, fulgurance de la révélation de la vraie voie, ces trois faits d'une narration d'une extraordinaire originalité en cette fin de $\mathrm{IV}^{\mathrm{e}}$ siècle sont devenus les armatures d'une construction narrative archétypale, $a$ Christian classic, au point que des siècles après, une Thérèse d'Avila pouvait s'y reconnaître exactement.

Mais ce qui rend également exemplaire l'aventure d'Augustin, laquelle a fait que Thérèse d'Avila y a trouvé des points de repère, c'est la structuration que sa narration fait de sa vie. Comme le souligne justement Karla Poewe ${ }^{23}$, le récit s'organise plus sur un contraste que sur une progression lente, sur un contraste entre le manichéen dans l'academia et l'évêque dans l'ecclesia. La conversion fracture la vie. Les événements vécus, les pensées émises, les émotions exprimées sont ainsi classées en fonction non pas de leur occurrence (à leur place dans le temps qui s'écoule) mais de la seule position que l'auteur leur donne, avant ou après le moment de la conversion. Une telle dichotomie renvoie - bien évidemment, pourra-t-on dire - à un jeu de symboles opposés, lesquels font partie de la culture actualisée (structurée en fonction de ses intérêts propres, de ses passions...) d'Augustin. Pour autant, Karla Poewe conteste l'opinion commune (qui semble être celle de M. Darrol Bryant, notamment) selon laquelle chaque étape philosophique de sa vie doit être prise comme un moment de sa conversion, car l'expérience philosophique n'est qu'intellectuelle et précisément incapable de changer la vie. Le changement de vie n'est pas une opération intellectuelle mais une expérience. Et c'est son expérience qu'observe Augustin. Il examine ses actes mondains pour y déceler des éléments de la sagesse de Dieu, c'est une opération métaphorique qu'il instruit avec des concepts théologiques; il décrit les moments forts de la conversion pour y déceler le plan divin, c'est une opération métonymique qui exige une reconstitution de faits d'émotion. La destinée de l'expérience augustinienne est donc double. Beaucoup privilégient l'expérience théologique d'Augustin, certains privilégient son expérience émotionnelle - c'est le cas des charismatiques d'aujourd'hui.

La conversion chrétienne se raconte. Elle est un drame qui casse une vie en deux, qui dispose un avant et un après, selon une suite de séquences qui semblent défiler dans une logique serrée jusqu'à l'identification de soi et la reconnaissance du sens du monde. Avec Augustin, le récit de conversion eut une sorte de naissance - naissance d'un récit mais aussi naissance d'un modèle, dans lequel Thérèse d'Avila mais aussi les charismatiques, mais aussi des écrivains et des artistes devenus chrétiens, peuvent reconnaître leur propre expérience. Le construit narratif de

${ }^{23}$ Karla Poewe, « Conversion among charismatics », Religious Conversion, pp. 191-206. 
la conversion apparaît comme un possible dans lequel on puise, selon ce que l'on vit et ce que l'on est, mais aussi comme une contrainte. Et il tire précisément sa puissance performative de son caractère de contrainte. C'est ce qu'il faut montrer maintenant: une contrainte dans la mesure où il mobilise des ressources d'ordre théologique.

\section{La conversion comme tradition}

Il faut parler d'histoire, non pas pour faire l'histoire de la conversion chrétienne, ni l'histoire de ses récits, mais pour entrevoir ce qui a constitué leurs traits les plus marqués. C'est-à-dire pour entrevoir ce que peut être la tradition de la conversion.

Il y a, selon Jean-Luc Blaquart ${ }^{24}$, plusieurs traditions de conversion, traditions qui se sont succédé au long du temps. Chaque tradition constitue une sorte de fonds culturel dans lequel les convertis, selon leur temps et leur intérêt, vont puiser ce qui leur sert de cadre de représentation de leur proche geste, à la fois comme fait de connaissance et comme cadre normatif. Une suite - non pas historique mais proprement théologique - des différentes traditions est ainsi proposée. La conversion comme retour serait, par excellence, la conversion de la tradition première, où l'acte est à la fois un retournement et un retour à un état ou à un modèle ancien; la conversion évangélique, qui est la rédemption dans le christianisme, serait le terme de cette suite. Entre la conversion comme retour et la conversion évangélique, les moments d'une méta-histoire du salut seraient la reconversion rationnelle (la metanoia), la conversion biblique (la rencontre du Dieu qui parle), la conversion gnostique. La conversion moderne enfin, comme un appendice malheureux, serait celle de l'homme qui se croit libre et refuse ce dont il est héritier, c'est-à-dire un monde de traditions.

Cette série, si elle était un continuum de représentation religieuse, comme semble le penser l'auteur, serait tout aussi téléologique que théologique : comme si la sédimentation logique des différentes traditions produisait ce cristal qu'est la dite conversion évangélique. Mais ce qui compte est le fait que la série est en quelque sorte ancrée dans une vision principielle de la conversion comme retour, alors que celle-ci est caractéristique de la conversion biblique, dans une mythique du retour au Dieu d'Israël après le dévoiement des cultes idolâtres, et alors qu'elle est totalement étrangère à la conversion comme metanö̈a ${ }^{25}$. Il reste que la question se pose : qu'est-ce qui fait que, dans une théologie chrétienne, soit posée au départ la conversion comme retour, comme retour au bercail ?

La contribution de Nancy Gauthier ${ }^{26}$ est un rappel à l'histoire. Les quelques mentions de conversion issues de l'Antiquité tardive sont de nature indirecte, elles ne sont jamais démonstratives, jamais circonstanciées. Le processus de conversion

\footnotetext{
24 Jean-Luc Blaquart, « Nos traditions de conversions. Approche philosophique et théologique », La conversion religieuse, pp. 17-25.

25 Christian DÉcobert, «La conversion comme aversion », Arch. 104, 1998, pp. 33-60.

${ }^{26}$ Nancy GAUTHIER, "Les récits autobiographiques de conversion sous l'empire romain », La conversion religieuse, pp. 27-44.
} 
religieuse n'est jamais décrit que par ses entours. Entours qui sont, dans un avant, un temps de recherche personnelle du vrai et du bien et, dans un après, un changement de comportement. Mais une histoire des modalités de cette recherche est possible (scandée par des noms tels que Justin, Hilaire de Poitiers, mais aussi Apulée, Julien l'Apostat), qui permet de saisir que les plus anciens lettrés convertis relatent, comme un passage obligé, un parcours du stoïcisme au platonisme, ou un parcours de l'accumulation des pratiques à mystères au culte unique à Isis. On comprend également que l'expansion du néo-platonisme fut ce moment nodal qui permit de penser - massivement, pourrait-on dire - le recouvrement du vrai par le bien et que c'est sur lui que les expériences du mal purent être articulées, jusqu'à l'ultime et en quelque sorte totale épreuve d'Augustin d'Hippone.

Résumons : la conversion d'Augustin est à la fois comme un point d'arrivée et comme un point de départ; quelque chose se construit jusqu'à Augustin, son récit n'est pas un météore venu d'un astre lointain, son récit est à la fois très singulier et très lisible, il renvoie à des catégories de pensée et à des symboles partagés, donc construits, donc hérités. Il y a, en effet, quelque chose d'hérité, de déjà convenu dans les Confessions. Mais quelque chose part, en revanche, des Confessions. Un récit nouveau est fixé, une narration est imposée, qui vont perdurer.

Changement d'époque, changement de milieu. La conversion des grands au Moyen-Âge occidental 27 élargit le champ des interrogations. C'est d'abord le processus de christianisation qu'elle permet d'entrevoir, puisque la conversion au christianisme se produit notamment par le haut et que donc le baptême d'un roi ou d'un duc produit en étoile des conversions qui correspondent à son aire de domination ou d'influence ${ }^{28}$. Mais les circonstances des conversions proprement dites des grands sont difficiles à saisir, à partir des chroniques qui ont été conservées. Car comment déchiffrer ce que l'on y lit? D'autant qu'il est aisé de constater que ces textes ont des schémas narratifs très topiques, notamment pour les faits de conversion, et qu'ils se ressemblent tous trop pour sembler véridiques. Comment comprendre ce qui est écrit du baptême de Clovis, pour prendre l'exemple le plus célèbre ? On a beaucoup parlé des possibles motivations de Clovis à devenir chrétien : était-ce par opportunisme politique, pour rallier à lui un puissant clergé ? Était-ce dans un état de crise post-traumatique (la mort prématurée de l'un de ses fils, baptisé de surcroît) ? Était-ce parce qu'il s'interrogeait sur la mort?

On peut développer longtemps chacune des trois réponses, et l'on ne s'en est pas privé. Mais on peut aussi se demander s'il y a une quelconque pertinence à s'engager de la sorte si des distinctions opératoires ne sont pas faites. Distinctions entre motivation, demande religieuse et offre religieuse. La motivation est du registre de l'action et de l'identification de soi, la demande et l'offre sont du registre de la représentation. En l'occurrence, l'intérêt propre (l'opportunisme politique) et le traumatisme (l'expérience de la mort) ne sont pas des réponses que l'on peut proposer en concurrence ou sur un même plan. La première est avancée par l'historien, la deuxième entre dans le récit de la conversion, elle est donc proposée

27 Jean Heuclin, « Les grands aristocrates mérovingiens et les enjeux de la conversion », La conversion religieuse, pp. 57-66.

${ }^{28}$ Voir, par exemple, Karl Ferdinand WERnER, « Le rôle de l'aristocratie dans la christianisation du Nord-Est de la Gaule », in Pierre Riché (dir.), La christianisation des pays entre Loire et Rhin (IVo-VII siècles), Paris, Cerf, 1993, pp. 45-73. 
par le biographe ou le chroniqueur ; la deuxième peut, pour l'historien, cacher la première, elle ne peut en tenir lieu dans la mesure où elle est emprise dans une représentation qui est celle du biographe ou du chroniqueur, et que celui-ci tend à faire partager. La motivation réelle de Clovis à devenir chrétien ne nous est pas connue et le récit de sa conversion par Grégoire de Tours ne nous aide guère à y voir clair. En revanche, ce récit entre dans un cadre théologique d'une grande précision.

Le récit repose, en effet, sur une série de glissements. Clovis est douloureusement frappé par la mort d'un fils : ce fort traumatisme fait qu'il s'interroge sur le monde qui l'entoure, sur les choses. Premier glissement: de l'expérience du traumatisme à la réflexion sur le sens des choses. De fait, les récits de conversion, anciens et modernes, rapportent à l'envi ces moments de doute profond et douloureux, où l'ordre des choses vacille, où les certitudes s'éloignent. L'interrogation sur la mort apparaît comme l'une des manifestations mêmes de ce doute. Deuxième glissement : de la réflexion sur le sens de ce monde-ci à un questionnement à propos de l'autre monde. Le questionnement sur la mort et sur ce qui vient après elle n'est que la manière suspendue d'espérer une vie éternelle, telle que précisément en parle le discours chrétien. Troisième glissement: d'un questionnement sur l'autre monde à une vision sur ce qu'il y a après la mort.

En trois glissements, le récit est passé d'une motivation supposée, un désordre ou un dérèglement personnel, à une demande religieuse et de la demande à une exposition de réponse. Dans le récit, quelque personnage ou quelque écrit est alors porteur de cette réponse. Nous retrouvons l'ossature supposée topique de la narration de la conversion chrétienne : crise, langage religieux, quête, rencontre.

Pour autant, la signification du récit est celle d'une transaction où une offre (la vie éternelle) est mise en contiguïté avec une demande éventuelle (un questionnement sur la mort). S'il y a une motivation (ce qui pousse à l'action) tangible et décelable (en l'occurrence un traumatisme : mort, maladie), il y a, pour lui faire face, un discours de l'offre. Le récit de la conversion est un procès qui tend à mettre en collusion les deux faits et à transformer la motivation en une quête, laquelle quête équivaudrait à une offre faite. Mais faite par qui ? Par les hommes autorisés de l'Église chrétienne, directement ou non. Cette offre est à la fois doctrinale et institutionnelle.

Un bel exemple de conversion, développé dans la même contribution ${ }^{29}$, nous éclaire sur les distinctions qu'il faut opérer entre une motivation, une demande et une offre institutionnelle. Le roi Edwin de Northumbrie ${ }^{30}$ est menacé de mort, en même temps qu'on le presse de se convertir au christianisme. Un homme l'informe qu'un complot se trame contre lui et lui dit également que s'il se convertissait au christianisme, cela lui ôterait toute angoisse à propos de son futur. Ébranlé, Edwin réunit bientôt un groupe de notables et les interroge sur cette nouvelle croyance. L'un des notables répond que la vie humaine sur terre est semblable à cet instant de vol d'un moineau dans une pièce qu'il traverse, d'une ouverture à l'autre. Sur ce qu'il y a eu avant cet instant, sur ce qu'il y aura après, l'homme ne sait rien. Si la nouvelle croyance en dit plus, si elle permet d'en savoir plus sur cet avant et sur cet

${ }^{29}$ Idem, pp. 59-60.

${ }^{30}$ L'histoire de la conversion d'Edwin de Northumbrie est importante dans la mesure où elle marque les débuts de la christianisation massive de l'Angleterre. 
après, c'est qu'elle est bonne. Après ces paroles, Edwin se convertit et autorise que l'on prêche le christianisme dans son royaume.

Nous retrouvons un procès narratif connu. Edwin est en danger de mort et l'opportunité de l'éloignement du danger lui est offerte sous forme de conversion, voilà pour la thématique générale. Plus précisément, et comme pour la conversion de Clovis, le danger que représente la mort (c'est-à-dire la crise) est double : il y a la mort qui s'approche, les hommes qui veulent assassiner Edwin ; il y a, par ailleurs et de façon plus permanente, une ignorance devant la mort qui rend celle-ci terrifiante; cette crise devient, dans une expression chrétienne (le langage religieux), une interrogation sur la mort et l'au-delà (la quête). Quelqu'un lui dit que s'il se convertit à la fois la mort s'éloignera et la terreur de la mort se dissipera ; puis l'un des hommes de son entourage lui signifie que le christianisme apporte une réponse à ce qui est pour lui inconnu (la rencontre).

Sur la motivation "vraie » d'Edwin à devenir chrétien, nous ne savons pas grand chose, sauf que les faits sont têtus et situent sa conversion dans un contexte d'alliance matrimoniale. Edwin devint chrétien quand il épousa la princesse catholique Aethelburth de Kent: Aetherburth emmena Paulin, archevêque d'York, en Northumbrie, lequel Paulin convertit Edwin en 627, puis nombre des dignitaires du royaume. Mais le présent récit n'en dit pas grand-chose. Il n'est question que de danger, de trahison, de mort qui s'approche. Une demande religieuse est ainsi formulée à propos de la mort et de l'au-delà, elle prend place de motivation, c'est la belle image de l'oiseau qui vient d'on ne sait où et qui va on ne sait où. Mais les paroles du notable, si elles contiennent une annonce implicite de vie éternelle, promettent surtout un savoir, elles sont l'annonce d'une offre. Toute l'histoire se rapporte à ce fait : au jeune roi, une offre chrétienne est faite, sous forme d'un savoir sur les choses - la vie après la vie est désormais quelque chose qu'il connaîtra et qui le fera triompher de la mort.

L'offre est dite par la bouche d'un notable, d'un compagnon du roi Edwin. Mais qui parle vraiment? Et quelle est la nature de ce récit? Le passage est extrait de l'Historia ecclesiastica gentis Anglorum, écrite par Bède le Vénérable. Bède (672-735) était moine, il passa toute son existence au monastère de Wearmouth (dans le Northumberland), il rédigea des textes théologiques et historiographiques. Inutile d'en dire beaucoup plus. Une Histoire ecclésiastique des Anglais, une Histoire des Anglais d'un point de vue religieux. Dans cette Histoire, un récit de conversion de roi est un morceau de choix dans la mesure où il a valeur exemplaire, et tout au long de son Histoire, Bède le clerc, l'homme autorisé, réalise un programme : il déploie un discours doctrinal. Dans l'expérience de la conversion d'un roi, qu'il se représente de façon très explicite comme une transaction, Bède fait entrer un élément de doctrine : c'est le savoir chrétien qui sauve, c'est l'expression du message du Christ. Ce qui apparaît comme l'offre dans la transaction de la conversion est explicitement un fait doctrinal.

L'on peut, au demeurant, retourner la proposition et avancer que l'un des vecteurs les plus efficaces de diffusion des éléments de la doctrine chrétienne devait être le corpus des biographies royales et des récits de soumission des princes au christianisme. Cette littérature royale, princière, où les conversions sont des moments éminents, puisqu'elles organisent une scansion dans une Histoire de l'installation du royaume de Dieu sur terre, furent de véritables supports d'une éducation partagée, populaire au sens où elle était diffusée largement, ne serait-ce que 
sous forme de sermons, de commentaires, de contes. Mais si ces histoires parlent d'offres, les doutes et les calculs - autrement dit, les motivations - d'un Clovis ou d'un Edwin de Northumbrie demeurent dans une ombre épaisse, presque impénétrable.

Au total, une tradition établie du récit de conversion met en relation de cause à effet une crise personnelle, un quelconque traumatisme ou un mal être, et une demande religieuse sur le sens des choses; comme elle met en équivalence une telle demande religieuse avec une offre religieuse. Mais si l'on renverse l'ordre des éléments du récit et que l'on suppose que dans le récit l'offre est une donnée première, qu'elle est posée dans un programme dogmatique, on pourra saisir toute la topique d'une telle narration. Laquelle narration a, par conséquent, toutes les chances de durer, le temps que dure le programme dogmatique.

\section{La conversion dans l'institution}

Loin du point de vue adopté par Jean-Luc Blaquart, il m’apparaît que la représentation historiquement première de la conversion, en tout cas celle de l'aube du christianisme, n'était certainement pas une action de retour mais une action de retournement; elle n'était pas une action où l'acteur revenait au bercail mais où il changeait de bercail en changeant brusquement ou progressivement de direction. L'exemple d'Augustin l'atteste, mais le cas «primitif » le plus évocateur est celui de Paul. À l'évidence, sa conversion au christianisme, telle qu'elle est rapportée par Luc, est brutale, totale, et elle fait entrer Saul, sous le nom de Paul, dans un monde qui lui était étranger. Et cette représentation de la conversion comme intégration dans un monde nouveau a perduré jusqu'à Augustin, et bien plus tard. D'autre part, nous avons vu que les récits de narration, d'Augustin à maintenant, se structuraient sur une crise, un traumatisme vécu, une situation de mal être... que l'engagement dans le christianisme dissipait ou tout au moins auquel l'engagement dans le christianisme donnait sens. À ce propos, le fait de «crise» est tellement prégnant que par excès de modélisation on le prendrait volontiers pour universel. Il est significatif, en effet, que Lewis R. Rambo et Charles E. Farhadian parlent de crise quant à la conversion de Paul et la font entrer dans le schéma narratif qu'ils élaborent, alors qu'évidemment elle échappe au modèle.

Il convient ainsi de rendre compte de l'apparition de la représentation de la conversion au christianisme comme structurée sur une crise, de même qu'il convient de rendre compte de l'apparition d'une représentation possible de la conversion au christianisme comme un retour à la vraie croyance. L'hypothèse que je formulerai rapidement, dans l'espace de cette note critique, est que les deux faits furent liés, et furent liés à un processus de mise en place institutionnelle.

Grossièrement dit, le christianisme est passé de l'état de «mouvement religieux » à l'état d' «'́glise », au sens troeltschien ${ }^{31}$, en se dotant d'institutions sacrales. La mise en chantier de telles institutions correspond d'abord à la distinc-

31 Jean SÉGuY, Christianisme et société. Introduction à la sociologie de Ernst Troeltsch, Paris, Cerf, 1980, pp. 101-111. 
tion de quelques gestes. Ces gestes sont des rites. Ces gestes eurent une valeur de reconnaissance entre ceux qui les pratiquaient, comme une valeur de connaissance : dans leur pratique, ceux qui les accomplissaient se situaient dans un certain rapport avec leur Dieu et avaient connaissance de la nature de ce rapport. Le caractère hiérophanique de tels gestes étant l'indice même de la réalité de cette connaissance. Des effets de système s'établirent entre les différents gestes rituels qui étaient cohérents entre eux, effets de système qui constituèrent des frontières et une armature et qui permettaient les distinctions entre les hommes : entre les vrais croyants et les autres, entre les vrais croyants eux-mêmes. Plus largement, la mise en place de telles institutions reposait sur l'identification nouvelle de normes. Une norme est la formulation de ce qui doit être respecté au nom d'une loi sacrée qui permet le jugement ; une norme chrétienne produisait ainsi une classe de gestes volontaires, gouvernés par le bien, par la soumission au message christique. Le respect de la norme reposait sur une autorité légitime, laquelle autorité, circulairement, n'existait et n'agissait que dans le cadre des valeurs définies pour qu'existât la norme chrétienne. Les hommes de l'autorité légitime, hommes de connaissance, pratiquèrent les rites, dirent le bien, diffusèrent le message christique. Une distinction était fixée entre eux, les clercs, et les autres, les profanes.

Concrètement, ce travail de production d'institutions sacrales fabriquait des frontières, en même temps qu'elle édifiait une théologie. Les frontières entre les vrais croyants et les autres étaient à la fois celles qui séparaient ces vrais croyants des non croyants (païens) et celles qui les séparaient des hérétiques. Situer l'hérétique était l'un des enjeux majeurs de la constitution d'une Église pensée comme orthodoxie et comme communauté ${ }^{32}$. L'hérétique était-il un homme dévoyé, un ennemi de Dieu, était-il un chrétien pécheur ou n'était-il plus chrétien ? Et l'hérétique repenti, rallié à l'orthodoxie, devenait-il chrétien, redevenait-il chrétien? L'hérésie était-elle un nouveau paganisme ou une dissidence ? Était-il un fait extérieur ou intérieur à l'Église ? On le voit, ces questions, qui ne cessaient de tarauder les esprits des penseurs qui se disaient orthodoxes, les obligeaient à penser un intérieur et un extérieur, à penser avec toujours plus d'acuité ce que pouvait être une frontière pour l'Église chrétienne.

D'autre part, la théologie chrétienne reposait notamment sur la croyance en une rédemption de l'homme ; et comme matériau, elle avait à brasser le corpus scripturaire des deux Testaments. Une pensée intertestamentaire émergeait, qui balisait une Histoire de salut: la révélation christique inaugurait une nouvelle alliance qui était à la fois une reformulation et un dépassement de l'ancienne alliance d'Abraham. Qui plus est, la pensée de la rédemption supposait celle de la faute originelle : la rédemption du vrai croyant équivalait pour celui-ci au retour à l'état d'avant la faute, à l'effacement de la faute. Et le baptême se voulait le rituel de la rémission de la faute dont chaque homme héritait, comme de la mort. C'est précisément au temps d'Augustin que la question du péché originel devint centrale : son œuvre polémique et théologique, De peccatorum meritis et remissione, sa lutte acharnée

32 Voir Alain Le Boulluec, La notion d'hérésie dans la littérature grecque, II -IIIe siècles, Paris, Institut d'études augustiniennes, 2 tomes, 1985. 
contre le pélagianisme contribuèrent à faire de l'héritage du péché originel un dogme central à la justification du christianisme ${ }^{33}$.

En somme, la nécessité de penser l'hérésie, comme plus globalement de penser un intérieur et un extérieur à l'Église, la nécessité de penser la rédemption, comme de penser une Histoire de salut dans une perspective intertestamentaire, étaient concomitantes. Elles convergèrent vers une représentation du passage au christianisme à la fois comme un dépassement et comme un retrait. Le retrait est le fait que l'on a retiré quelque chose à une entité, ou que quelque chose est tiré en arrière ou de côté; ce n'est pas un retour, qui serait simplement le fait de repartir à l'endroit d'où l'on vient, c'est bien plutôt l'action - et la mémoire vive - de se débarrasser ou d'être débarrassé de quelque chose ou l'action d'abandonner un lieu. Dans la vision du passage au christianisme qui se fabriqua aux $\mathrm{IV}^{\mathrm{e}}$ et $\mathrm{V}^{\mathrm{e}}$ siècles passage au christianisme dans l'universel, dans une Histoire de salut, mais aussi pour chacun des croyants - il y eut cette connotation forte de contraction, de réduction, d'un quelque chose ou d'un quelque part qui était laissé. Cette connotation forte a imprégné les récits de conversion qui alors se multipliaient et se fixaient dans une narration obligée.

La littérature de la conversion religieuse fut d'abord comme une sous-catégorie de la littérature des martyrs, qui prit, à partir du $\mathrm{IV}^{\mathrm{e}}$ siècle mais surtout au $\mathrm{V}^{\mathrm{e}}$ et au VI ${ }^{\mathrm{e}}$ siècles, une importance considérable dans le monde chrétien. Véhiculée par des sermonaires, cette littérature édifiante fixait dans des évocations flamboyantes un culte des martyrs qui s'installait dans les terres christianisées, en même temps qu'elle produisait une histoire des premiers temps, dans la persécution, de la vraie religion. Les histoires de martyres étaient pleines de récits de conversions.

Sans pouvoir ici développer par trop ce point, je dirai seulement que cette « martyrologie triomphante" reposait sur la procédure narrative de la passion épique, dont le modèle évident était la passion du Christ ${ }^{34}$. Les histoires de martyrs, très stéréotypées, sont construites sur une série de séquences qui sont globalement les suivantes: l'action se situe au temps de la persécution de l'empereur (Dioclétien, généralement, bien qu'il ne soit pas toujours nommé) contre les chrétiens. Un jeune homme, soit de noble allure soit de noble famille, rallie la nouvelle religion. L'affaire est vite connue et il est présenté devant le Gouvernant - le représentant de l'empereur ou l'empereur lui-même. Celui-ci use de tous les instruments de la persuasion pour lui faire abandonner sa foi, le flattant et lui proposant une haute fonction, le menaçant et lui décrivant les mille tourments ; la joute publique entre le Gouvernant et le converti se fait très longue, d'autant que le Gouvernant est peu à peu mis en difficulté par la superbe du néophyte chrétien. Le Gouvernant ordonne enfin la torture puis la mort du converti. Dans une passion tout aussi interminable, le converti survit miraculeusement à plusieurs mises à mort, soutenu par les visions du Christ ou les messages de l'Archange qui lui promettent une prochaine félicité. Sa mort est suivie par une reconnaissance rapide de sa sainteté et par un culte naissant à ses pieuses reliques. En résumé, l'objet d'une telle littérature

33 Athanase SAGE, «Le Péché originel. Naissance d'un dogme », Revue des études augustiennes 13, 1967, pp. 211-248; idem, «Le Péché originel dans la pensée de St. Augustin de 412 à 430 », Revue des études augustiniennes 15, 1969, pp. 75-112.

${ }^{34}$ Hippolyte Delehaye, Les Passions des martyrs et les genres littéraires, Bruxelles, Société des Bollandistes, 1921 (rééd. 1966). 
est de désigner la concomitance entre le martyre et la diffusion du christianisme c'est la fonction du martyre que d'être le mobile de la christianisation.

$\mathrm{Au}$ long de sa courte vie de chrétien, quand il est encore libre ou quand il est déjà entravé, le saint-converti provoque les conversions de personnes qu'il rencontre. Les récits de ces conversions ont un développement tout aussi stéréotypé : faute - châtiment - rencontre - guérison - conversion - engagement. Telle personne a accompli tel acte mauvais ou est possédé par le démon. La conséquence de cet acte ou de cet état est que la personne elle-même mais plus souvent l'un de ses proches (le fils, l'épouse) tombe gravement malade. Désespéré, il cherche à s'approcher du saint (le futur martyr) dont il a entendu parler et qui, aux dires de tous, accomplit des miracles. Le rencontrant, il lui demande de le guérir, ou de guérir son fils ou son épouse. Un dialogue s'engage au cours duquel, dans un premier temps, le saint propose à l'homme de renoncer à sa faute, auquel cas il le guérira, guérira le fils ou l'épouse - et l'on apprend que la vraie faute de l'homme en question n'est pas ce que l'on avait compris au début du récit (une action mauvaise, la possession par le démon), mais qu'elle est religieuse, car l'homme est un impie, il sacrifie aux dieux, ou il persécute les chrétiens. Mais l'homme retourne la proposition du saint et déclare que si la guérison survient, alors il deviendra chrétien. La guérison survient et l'homme devient chrétien. Son engagement de chrétien est ensuite signalé par un nom qu'il porte ostensiblement, par une exhortation aux gens de se convertir, etc.

Mais un tel schéma narratif de conversion ne servit pas seulement la littérature martyrologique. Il fut ensuite reproduit pour raconter les conversions accomplies par des saints, non martyrs, de même qu'il servit à raconter les procédures de réintégration dans l'orthodoxie, au contact d'un homme pieux généralement, des hérétiques.

Dans le récit ancien d'une conversion au christianisme, il y a, pour résumer, une faute première. L'ignorance première de Dieu, ou l'hérésie première, sont un décalque du péché originel. Puisque la maladie est une forme communément reconnue, dans le monde ancien et médiéval, d'interférence du monde suprasensible dans le monde sensible, elle est en l'occurrence comprise comme un châtiment divin qui frappe l'homme pécheur, comme la mort d'un proche ou la menace de mort. La rencontre du pécheur avec le saint homme (qui est le chrétien par excellence) est le déclencheur de la rédemption du pécheur - l'effacement, mais non l'oubli, du péché originel - tout en étant l'instance de connaissance par le pécheur de son péché. L'explication est ainsi mêlée à une véritable transaction. L'homme fautif et châtié demande au saint qu'il lui assure la guérison ou l'éloignement de la mort en préalable à sa conversion, ce qui veut dire qu'il pose de façon explicite l'intérêt qu'il a hic et nunc à se convertir. L'explication du péché est également un discours de l'offre religieuse (offre de rémission de la faute, de cessation du châtiment et de salut), mais où l'offre faite dans un premier temps se retourne en demande dans un second temps.

Cette construction narrative fut stable. On pourrait la suivre au long de l'histoire du christianisme, mais une note critique n'est pas un essai. À mesure que la christianisation s'achevait, en Orient comme en Occident, les conversions d'hérétiques, ou d'hommes dissolus (restés païens dans leur âme), prenaient le pas sur celles des masses païennes. Les saints médiévaux souvent guérissaient et convertissaient dans le même geste. 
Les contributions « historiennes » des deux ouvrages, Religious Conversion et La conversion religieuse, montrent en définitive, dans leur rapprochement avec des contributions sur le monde contemporain, qu'il y a un lien génératif, en quelque sorte, entre un temps de construction institutionnel (le temps d'Augustin, de l'invention du péché originel, de l'aménagement des démarcations face au territoire de l'hérétique), les temps médiévaux de réalisation de la christianisation (le temps de la conversion des princes et de la distinction des clercs) et les temps modernes. Ce lien est, quant à la conversion, celui qui s'est établi entre l'invention et la reproduction obligée d'une narration conçue comme un exposé dogmatique, où une histoire personnelle et conventionnelle à la fois rejoint l'Histoire de salut. Et si l'histoire de la conversion au christianisme était instituée, c'est bien parce que l'institution conférait son identité au chrétien ${ }^{35}$. Il n'y a pas que les lieux sacrés des communautés religieuses qui sont nécessaires et dogmatiques ${ }^{36}$, qui sont reproduits tant que durent ces communautés, il y a aussi les récits anciens, ceux qui distinguent entre les hommes et indiquent la voie de la vraie croyance.

Le récit de la conversion a évolué avec les siècles. Peu à peu la maladie, comme symptôme aigu de la crise, s'est éloignée. L'ont remplacée le désordre des sens et la magie, et l'on sait à quel point les affaires de sorcellerie et de possession étaient à l'âge moderne liées à des reconnaissances d'apostasie et des procédures de reconversion. Quant à la représentation de la crise comme mal être, comme indéfinissable mal à l'âme, elle est antérieure à la guerre religieuse de la fin du $\mathrm{XIX}^{\mathrm{e}}$ siècle. Le René de Chateaubraind, inséré en 1802 dans Le génie du christianisme, inaugurait en littérature cette thématique du mal à l'âme comme mobile à un parcours de conversion, laquelle thématique fera bientôt fortune en littérature (l'on peut d'ailleurs se demander pourquoi Chateaubriand, l'initiateur, n'est pas présenté parmi les littérateurs dans l'ouvrage dirigé par Emmanuel Godo).

Il faut enfin conclure. Par un retour à l'enquête de Amazing Conversions sur les nouveaux convertis et les nouveaux apostats. Les figures symétriques des Amazing Believers et des Amazing Apostates sont prises par les enquêteurs pour ce qu'elles ne sont certainement pas: les postures ingénues de jeunes gens à la recherche d'eux-mêmes. La figure du converti est traditionnelle, instituée (et celle de l'apostat, on l'a vu, est son symétrique imparfait). Elle fait partie de ce stock disponible, inactualisé, que constitue ce qu'on appelle la culture chrétienne, ou le capital chrétien de symboles et de mythes. Mais surtout, la figure est réapprise par ces jeunes gens. Parce qu'après avoir été seulement convenue, emprise dans une convention dormante, elle renvoie pour eux à quelque chose de réel, de parlant. Car ce qui est bien réel est, après les dommages subis par la forteresse chrétienne, la multiplication des offres religieuses, où le christianisme n'apparaît plus que comme l'une des offres possibles, et où l'incroyance, le déni de la croyance, le changement de croyance semblent être au chrétien insatisfait des voies désormais ouvertes.

${ }^{35}$ L'institution ne décrète pas seulement l'identité des choses, elle contribue également à donner aux gens leur identité. Marie Douglas, Comment pensent les institutions, Paris Éditions du Mauss, rééd. 1999, pp. 73-84.

36 Maurice Halbwachs, La topographie légendaire des Évangiles en Terre sainte. Étude de mémoire collective, Paris, Presses Universitaires de France, 1941 (rééd. 1971). 
Ce qui paraît réel est une ouverture du choix religieux. Les figures convenues du choix, bien enfouies dans la masse des figures disponibles du christianisme, réapparaissent alors, vivant une nouvelle vie. Puisqu'il y a choix religieux, ou apparence d'un choix immédiat à l'homme inquiet, la figure du converti est redevenue actuelle, vraie et mobilisatrice pour ceux qui les adoptent. Il y a une opportunité nouvelle à vivre le récit de la conversion.

Mais l'effet le plus paradoxal, peut-être, de la reconstitution de la conversion (comme une reconstitution judiciaire : des personnes refont ce que d'autres sont sensés avoir exactement fait) est l'exacerbation de l'exemplarité de la figure du converti. En effet, plus la promesse religieuse du salut semble s'évanouir, plus en fait la réalisation du royaume de Dieu semble s'éloigner, plus - en compensation semble s'imposer "la figure de l'individu régénéré qui témoigne personnellement de la puissance de transformation attachée à ce message $\gg{ }^{37}$. Mais la reconstitution n'est que partielle - la prégnance de l'institution chrétienne qui scelle le récit de conversion dans une dogmatique n'y est plus. Les enquêteurs de Amazing Conversion notaient, nous l'avons vu, que les «étonnants croyants » étaient largement incapables de donner une quelconque expression du salut qui leur était offert dans le groupement chrétien qu'ils rejoignaient. Sauf à dire qu'une certaine protection leur était assurée...

\author{
Christian DÉCOBERT \\ Centre d'Études Interdisciplinaires des Faits Religieux \\ CNRS-EHESS - Paris
}

${ }^{37}$ Danièle Hervieu-LÉGer, Le pèlerin et le converti, op. cit., pp. 146-147. 
